\title{
Radix Stellariae extract prevents high-fat-diet-induced obesity in C57BL/6 mice by accelerating energy metabolism
}

\author{
Yin Li ${ }^{1}$, Xin Liu ${ }^{1}$, Yu Fan ${ }^{1}$, Baican Yang ${ }^{\text {Corresp.. }}{ }^{1}$, Cheng Huang ${ }^{\text {Corresp. } 1}$ \\ ${ }^{1}$ School of Pharmacy, Shanghai University of Traditional Chinese Medicine, Shanghai, China \\ Corresponding Authors: Baican Yang, Cheng Huang \\ Email address: bcy2002@sina.com, chuang@shutcm.edu.cn
}

Stellaria dichotoma L., is widely distributed in Ningxia and surrounding areas in northwestern China. Its root, Radix Stellariae (RS), has been used in herbal formulae for treating asthenic-fever, infection, malaria, dyspepsia in children and several other symptoms. This study investigated whether the RS extract (RSE) alleviates metabolic disorders. The results indicated that RSE significantly inhibited body weight gain in high-fat (HF)-diet-fed C57BL/6 mice, reduced fasting glucose levels, and improved insulin tolerance. Moreover, RSE increased the body temperature of the mice and the expression of uncoupling proteins and peroxisome proliferator-activated receptors in the white adipose tissue. Thus, RSE alleviated metabolic disorders in HF-diet-fed C57BL/6 mice by potentially activating UCP and PPAR signaling. 


\section{Radix Stellariae extract prevents high-fat-diet-induced obesity in 2 C57BL/6 mice by accelerating energy metabolism}

3 Yin Li, Xin Liu, Yu Fan, Baican Yang*, Cheng Huang*

4 School of Pharmacy, Shanghai University of Traditional Chinese Medicine, 1200 Cailun Road, 5 Shanghai 201203, China

$7 *$ Corresponding author:

8 1. Cheng Huang, Drug Discovery Lab, School of Pharmacy, Shanghai University of Traditional 9 Chinese Medicine, 1200 Cailun Road, Shanghai 201203, China (chuang@shutcm.edu.cn). Tel: 10 +86-21-51322182; fax: +86-21-51322193.

11 2. Baican Yang, School of Pharmacy, Shanghai University of Traditional Chinese Medicine, 121200 Cailun Road, Shanghai 201203, China (bcy2002@sina.com). 


\begin{abstract}
Stellaria dichotoma L., is widely distributed in Ningxia and surrounding areas in northwestern
ABSTRACT China. Its root, Radix Stellariae (RS), has been used in herbal formulae for treating asthenicfever, infection, malaria, dyspepsia in children and several other symptoms. This study investigated whether the RS extract (RSE) alleviates metabolic disorders. The results indicated that RSE significantly inhibited body weight gain in high-fat (HF)-diet-fed C57BL/6 mice, reduced fasting glucose levels, and improved insulin tolerance. Moreover, RSE increased the body temperature of the mice and the expression of uncoupling proteins and peroxisome proliferator-activated receptors in the white adipose tissue. Thus, RSE alleviated metabolic disorders in HF-diet-fed C57BL/6 mice by potentially activating UCP and PPAR signaling.
\end{abstract}

\title{
INTRODUCTION
}

Metabolic syndrome (MS) is prevalent world-wide, particularly in Western countries. It is characterized by obesity, insulin resistance, hyperlipidemia, type 2 diabetes mellitus, hypertension, and atherosclerotic cardiovascular disease (Morikawa et al., 2004; Eckel et al., 2010). Excessive calorie intake and lack of exercise are the two main reasons leading to MS. Recent relevant experimental and clinical research results can be summarized as follows: (1) develop practical methods to address the main causes of MS, and (2) identify a direct method to eliminate adverse factors, such as insulin resistance, hyperlipidemia, obesity, and hypertension (Ginsberg, 2003). Thus, although difficult, novel therapeutics to prevent and treat obesity are urgently required (Apovian et al., 2015).

Brown adipose tissue (BAT) is essential for thermogenesis and body temperature maintenance (Harms \& Seale, 2013). When activated, BAT can express uncoupling protein1(UCP1) to release energy in the form of heat by uncoupling the protons generated by substrate oxidation during adenosine triphosphate (ATP) production (Izzi-Engbeaya et al., 2015). Moreover, white adipose tissue (WAT) can be used as an index of energy metabolism for it browning. UCP1, UCP2, and UCP3 are related to energy metabolism; in particular UCP1 plays a critical role in releasing electrons rather than storing them, resulting in heat release (Kim \& Plutzky, 2016).

Peroxisome proliferator-activated receptors (PPARs) are members of the nuclear receptor family (Francis et al., 2003). PPAR $\alpha$, PPAR $\beta$, and PPAR $\gamma$ are their three isoforms. PPARs are crucial regulators of lipid, glucose and tissue metabolism as well as cell differentiation and proliferation, apoptosis, and host immunity (Desvergne \& Wahli, 1999). PPARs bind to the retinoid $\mathrm{X}$ receptors to form heterodimers, which regulate downstream gene expression by interacting with PPAR response elements in these genes (Wahli, Braissant \& Desvergne, 1995). 
PPAR $\alpha$ is present in the liver (Jia et al., 2003), heart (Barger \& Kelly, 2000; Gilde \& Van Bilsen, 2003), skeletal muscle, BAT and kidneys. It mainly mediates the uptake and $\beta$-oxidation of fatty acids in the liver and heart (Bishop-Bailey, 2000; Puddu, Puddu \& Muscari, 2003). The activation of PPAR $\alpha$ is an effective therapy for hyperglyceridemia. PPAR $\gamma$ is expressed abundantly in adipocytes, particularly in WAT, as well as in the gastrointestinal tract and macrophages (Thompson, 2007). It plays a key role in adipocyte differentiation, lipid accumulation, and insulin sensitivity (Spiegelman, 1998; Francis et al., 2003), and is involved in whole-body glucose homeostasis (Barroso et al., 1999). As we all known, PPAR $\gamma$ is the target of the insulin-sensitizing agent rosiglitazone.

Radix Stellariae (RS), also called Yinchaihu, the root of Stellaria dichotoma, a common Chinese herbal medicine used clinically to treat fever and infantile malnutrition. RS was first described in "Ben Cao Gang Mu" 400 years ago (Teng, 1985). According to the clinical studies, RS also has several other pharmacological functions, including anti-inflammatory (Chen et al., 2010), anti-cancer, and anti-allergic activities (Morikawa et al., 2004; Sun et al., 2004) as well as dilation of blood vessels (Morita et al., 2005). Recently, it has been reported that RS had a higher content of $\alpha$-spinasterol, which has anti-inflammatory and antipyretic effect and $\beta$ carboline alkaloids in RSE with anti-allergy properties through the mice anti-allergic reaction experiment (Morikawa et al., 2004). Besides, new cyclicpeptids extracted from RS has been demonstrated with antitumor activity in vitro and mild dilation of blood vessels. Nevertheless, the effects of RS on metabolic disorders have not been reported.

In this study, we observed the effects of RSE on HF-diet-induced obesity to assay whether RS could alleviate metabolic disorders. We found that it can alleviate MS by reducing body weight and blood glucose levels, increasing insulin sensitivity in HF-diet-induced obese mice.

\section{MATERIALS AND METHODS}

\section{Preparation of Radix Stellariae extract}

RS was purchased from the Ningxia province. In a spherical extractor, $4 \mathrm{~L}$ of $95 \%$ ethanol was added to $500 \mathrm{~g}$ of RS. Extraction was performed for $2 \mathrm{~h}$ at $85^{\circ} \mathrm{C}$, followed by cooling and filtering of the extract. Extraction was repeated using 50\% ethanol. The extracted solutions were combined for rotatory evaporation at $60^{\circ} \mathrm{C}$, under reduced pressure, till the taste of alcohol was undetectable. Finally, the concentrated solution was freeze-dried and stored at $-20^{\circ} \mathrm{C}$.

\section{Liquid chromatograph-high resolution mass spectrometry}

Liquid chromatography-high resolution mass spectrometry (LC-HRMS) was performed using a Waters ACQUITY UPLC system, equipped with a binary solvent delivery manager and a sample manager. This system was, coupled with a Waters Micromass Q-TOF Premier Mass Spectrometer, equipped with an electrospray interface (Waters Corporation, Milford, MA). 
120

121

122

123

124

125

126

127

128

129

130

131

132

133

134

135

136

137

138

139

140

141

142

143

144

145

146

147

148

149

150

151

152

153

154

155

156

157

158

159

160

An Acquity BEH C18 column (100 mm × $2.1 \mathrm{~mm}$; i.d., $1.7 \mu \mathrm{m}$; Waters, Milford, USA) was maintained at $50{ }^{\circ} \mathrm{C}$ and eluted with gradient solvent from $\mathrm{A}: \mathrm{B}(95: 5)$ to $\mathrm{A}: \mathrm{B}(0: 100)$ at a flow rate of $0.40 \mathrm{~mL} / \mathrm{min}$, where $\mathrm{A}$ is aqueous formic acid $(0.1 \%(\mathrm{v} / \mathrm{v})$ formic acid) and $\mathrm{B}$ is acetonitrile $(0.1 \%(\mathrm{v} / \mathrm{v})$ formic acid) with an injection volume of $5.0 \mu \mathrm{L}$. The following gradient was applied (0 - $4.00 \mathrm{~min}: 95.0 \% \mathrm{~A}+5.0 \% \mathrm{~B} ; 4.00-6.00 \mathrm{~min}: 80.0 \% \mathrm{~A}+20.0 \% \mathrm{~B} ; 6.00-8.00$ min: $75.0 \% \mathrm{~A}+25.0 \% \mathrm{~B} ; 8-12.5$ min: $50.0 \% \mathrm{~A}+50.0 \% \mathrm{~B} ; 12.5-13.5 \min : 15.0 \% \mathrm{~A}+85.0 \%$ B; 13.5 - 15 min: $0.0 \% \mathrm{~A}+100.0 \% \mathrm{~B})$.

The MS analyses were performed using positive and negative ions channels. The ionization conditions were optimized, and the operating parameters were as follows: Polarity: positive/ negative; Capillary voltage: $3.0 \mathrm{kV} / 2.8 \mathrm{kV}$; Sampling cone: $35 \mathrm{~V} / 45 \mathrm{~V}$; Collision energy: $3 \mathrm{eV} / 3$ $\mathrm{eV}$; Source temperature: $115^{\circ} \mathrm{C} / 115^{\circ} \mathrm{C}$; Desolvation temperature: $350^{\circ} \mathrm{C} / 350^{\circ} \mathrm{C}$; Desolvation gas: $600 \mathrm{~L} / \mathrm{hr} / 600 \mathrm{~L} / \mathrm{hr}$; Scan range: m/z $50-1500 / \mathrm{m} / \mathrm{z} 50$ - 1500; Scan time: $0.3 \mathrm{~s} / 0.3 \mathrm{~s}$; Interscan time: $0.02 \mathrm{~s} / 0.02 \mathrm{~s}$.

\section{Animals and diets}

Six-week-old female C57BL/6 mice were purchased from the SLAC Laboratory (Shanghai, China). The animal protocols used in this study were approved by the Shanghai University of Traditional Chinese Medicine (approval number 2014019). The mice were housed under $22^{\circ} \mathrm{C}-$ $23^{\circ} \mathrm{C}$ with a $12 \mathrm{~h}$ light/dark cycle. After a 1 week adaptation period, we randomly divided the seven-week-old mice into three groups. They were fed a chow diet (Chow, $10 \%$ of calories derived from fat, Research Diets; D12450B), an HF diet (HF, 60\% of calories derived from fat, Research Diets; D12492), or a diet supplemented with 1\% RSE (HF + RSE). The mice had free access to food and water for 8 weeks. We weighed the food intake and body weight every 2 days.

\section{Rectal temperature measurement}

At the end of 8 weeks, the rectal temperature of the mice was recorded three times at 3 PM by using an instrument for measuring rectal temperature, at intervals of 2 days.

\section{Intraperitoneal glucose tolerance test}

All mice were fasted for $12 \mathrm{~h}$ overnight at the end of the preventive experiment. For the intraperitoneal glucose tolerance test (IPGTT), we collected the blood samples from the tail vein for determination of baseline glucose values $(0 \mathrm{~min})$. Next intraperitoneal injections of glucose ( $1 \mathrm{~g} / \mathrm{kg}$ body weight) were administered to all the mice in $15 \mathrm{~min}$, and blood glucose levels were measured at regular intervals $(15,30,60$, and $90 \mathrm{~min})$ after the injection of glucose.

\section{Intraperitoneal insulin tolerance test}


161

162

163

164

165

166

167

168

169

170

171

172

173

174

175

176

177

178

179

180

181

182

183

184

185

186

187

188

189

190

191

192

193

194

195

196

197

198

199

200

201

The mice were not fasted for the intraperitoneal insulin tolerance test (IPITT). Similar to the IPGTT, the basal blood glucose levels $(0 \mathrm{~min})$ were measured from the tail vein before the injection of insulin $(0.75 \mathrm{U} / \mathrm{kg}$ body weight). The insulin was diluted in physiological saline. Next, additional blood glucose levels were measured at 15, 30, 60, 90, and 120 min after the injection of insulin.

\section{Serum chemistry analysis}

The mice were fasted for $10 \mathrm{~h}$ overnight at the end of the animal preventive experiment; the next day, all mice were anesthetized using 20\% urethane before sample and tissue collection. Blood samples were drawn from the heart using a $1 \mathrm{~mL}$ syringe. After clotting at room temperature for over $2 \mathrm{~h}$, the serum was separated from the blood samples. After centrifugation, $120 \mu \mathrm{L}$ of serum was drawn from every sample, and the serum total cholesterol (TC), triglyceride (TG), lowdensity lipoprotein cholesterol (LDL-c), high-density lipoprotein cholesterol (HDL-c), alanine aminotransferase (ALT) and aspartate aminotransferase (AST) levels were analyzed using a Hitachi 7020 Automatic Analyzer.

\section{Morphological analysis of white adipose tissue}

To examine the structure of WAT, the WAT samples were fixed in $4 \%$ paraformaldehyde. The tissue samples were sectioned at $5 \mu \mathrm{m}$ intervals and stained with Hematoxylin and Eosin $(\mathrm{H} \&$ E). The stained samples were examined under a light microscope.

\section{Reporter assay}

The reporter assay has been performed using the Dual-Luciferase Reporter Assay System (Promega, USA) as previously described. The expression plasmids for pCMXGal-hPPAR $\alpha, \beta, \gamma$ and the Gal4 reporter vector MH100 × 4-TK-Luc were co-transfected with a reporter construct so that $1 \mu \mathrm{g}$ of the relevant plasmid combined with $1 \mu \mathrm{g}$ of reporter plasmids and $0.1 \mu \mathrm{g}$ of $\mathrm{pREP} 7$ (Renilla luciferase) reporter could be used to normalize transfection efficiencies. The transfection mixture, which contained $10 \mu \mathrm{g}$ of total plasmids and $15 \mu 1$ FuGENE®HD per ml of DMEM, was added to HEK293T cells for $24 \mathrm{~h}$ and then removed. The PPAR $\alpha, \beta, \gamma$ agonists (Fenofibric acid, GW7647, Pioglitazone) and 2.5, 5, 10, 20, 50, 100, 200, 400, 600, 800, $1000 \mu \mathrm{g}$ $/ \mathrm{ml}$ of RSE were added to fresh media and the cells were incubated for another $24 \mathrm{~h}$ to determine luciferase activity.

\section{Quantitative real-time polymerase chain reaction (Real time qPCR)}

The total RNA of WAT and BAT was extracted using the RNAiso Plus (Takara, Dalian, China). RNA is unstable, and to facilitate stable long-term preservation, we used the RevertAid First 
202 Strand cDNA Synthesis Kit (Thermo Scientific, Wilmington, Delaware, USA) for the first-strand 203 cDNA $\left(42^{\circ} \mathrm{C}, 1 \mathrm{~h} ; 70^{\circ} \mathrm{C}, 5 \mathrm{~min}\right)$. The gene expression levels were analyzed using quantitative 204 real-time RT-PCR conducted using the ABI StepOnePlus real-time PCR system (Applied 205 Biosystems, USA). The relative primers involved in the experiments are listed in Table 1. $\beta$ 206 Actin was considered an internal control to normalize the expression levels of genes. The cDNA

207 208

209

210

211

212

213

214

215

216

217

218

219

220

221

222

223

224

225

226

227

228

229

230

231

232

233

234

235

236

237

238

239

240

241

242

was denatured at $95^{\circ} \mathrm{C}$ for $10 \mathrm{~min}$ followed by 40 cycles of PCR $\left(95^{\circ} \mathrm{C}\right.$ for $15 \mathrm{~s}, 60^{\circ} \mathrm{C}$ for $\left.60 \mathrm{~s}\right)$.

\section{Statistical analysis}

Data were analyzed using SPSS 18.0, and the results were presented as mean \pm SEM. Differences were considered significant if $\mathrm{P}<0.05$. Statistical analysis included one-way analysis of variance, the Student's t-test, the Kruskal - Wallis H Test, and repeated measures analysis of variance.

\section{RESULTS}

\section{LC - HRMS detection of the main chemical contituents}

We performed the LC - HRMS assay to characterize the constituents in the extract. Ten compounds were putatively identified in the extract according to a previous report (Chen et al., 2010). We detected some $\beta$-carboline alkaloids, such as stellarine A - C, dichotomine B, $\mathrm{H}$, and L, glucodichotomine B as well as vanillin, 5-hydroxymethylfurfural (Fig. 1 and Table 2).

\section{RSE inhibited body weight gain in C57BL/6 mice induced by high-fat diet}

To investigate the effect of RSE on body weight gain, we selected the most widely used inbred strain C57BL/6 mouse, which is susceptible to diet-induced obesity, type 2 diabetes and atherosclerosis (Sun et al., 2016; Xia et al., 2016). The dose of RSE was determined by using a series of complex mathematical operation according to the dose of humans (15 g natural plant medicine/60 kg body weight/day) and pharmacology of traditional Chinese medicine (Zhang, 2002). The C57BL/6 mice were fed on a chow diet, HF diet or HF diet supplemented with $1 \%$ RSE for 8 weeks. The data revealed that the average body weight in the HF group was significantly higher than that of the Chow group (Fig. 2A), whereas the body weight of the HF + RSE group was evidently lower than that in the HF group from Week 2 to Week 8 (Fig. 2A). The data indicated that RSE could inhibit body weight gain induced by HF-diet in mice. There was no significant difference of food intake between the HF and HF + RSE groups (Fig. 2B). Furthermore, we observed the weekly food intake of the mice. The weekly intake of the three groups remained in a state of equilibrium (Fig. 2C). Thus, the lower body weight in RSE-treated mice was not caused by a lower calorie intake.

Next, we measured the adipocyte size using the H \& E stain. The results revealed that the size 
243

244

245

246

247

248

249

250

251

252

253

254

255

256

257

258

259

260

261

262

263

264

265

266

267

268

269

270

271

272

273

274

275

276

277

278

279

280

281

282

283

of WAT in the HF group was considerably larger than that of the Chow group, and RSE treatment reduced the size of WAT in HF + RSE group (Fig. 2D-2E). The data supported the conclusion that RSE inhibits body weight gain.

Inhibition of lipid absorption in the intestine or increase of energy expenditure may result in weight reduction. To test whether RSE affected the lipid absorption and energy expenditure, we determined the total cholesterol and triglyceride content of the feces of the mice, and measured rectal temperature. The RSE-treated mice did not exhibit an increase in the TC and TG content of feces (Fig. 2F-2G); however, the body temperature of the RSE-treated mice was notably higher than that of the mice in the other groups (Fig. 2H). Hence, an increase in energy consumption, and not the inhibition of intestinal lipid absorption, may be responsible for the reduction in body weight in HF + RSE group mice.

\section{RSE reduced fasting blood glucose and ameliorated insulin tolerance in high-fat diet- induced obese C57BL/6 mice at 15 and 30 min}

Obesity is a cause for insulin resistance and type II diabetes. Therefore, we measured the fasting blood glucose levels and glucose tolerance in the mice (Fig. 3A). RSE- treated mice exhibited lower fasting glucose levels than did the HF-fed mice (Fig. 3B). However, the blood glucose levels did not change following intraperitoneal injection of glucose. Then we tested the insulin tolerance in the mice. The results revealed that the blood glucose levels of the HF + RSE group were lowered than those of the HF group at 15, and $30 \mathrm{~min}$ (Fig. 3C).

\section{RSE lowered the lipid profile in serum and liver}

Obesity may be accompanied by hyperlipidemia. Therefore, we measured the lipid levels in serum and liver tissue. The fasting serum TG, TC, and LDL-c levels of the HF + RSE group were slightly lower than those of the HF group, although the levels were not significantly different between both groups (Fig. 4A). Similarly, the hepatic TG and TC levels were also lower in the RSE-treated mice (Fig. 4B). Then, we tested the serum levels of ALT and AST, the two indicators of liver damage. The levels of ALT of the HF group were evidently higher than those of the Chow diet-fed mice, indicating potential damage to liver function. RSE treatment, however, did not change the ALT and AST concentrations in HF group mice (Fig. 4C-4D).

\section{RSE induced the expression of metabolic gene in vivo}

The genes for uncoupling proteins (UCPs), namely UCP1, UCP2, and UCP3, are closely related to energy metabolism. In view of the rise of body temperature, we tested the expression of these genes in BAT, which participates in energy consumption and heat production. The expression of the genes in the RSE-treated mice BAT was not notably different from that of the HF-fed mice (Fig. 5A). White beige fat, indicated by high UCP1 expression (Nedergaard \& Cannon, 2014), which increases energy metabolism, may also be a mechanism of fat reduction. Therefore, we 
284

285

286

287

288

289

290

291

292

293

294

295

296

297

298

299

300

301

302

303

304

305

306

307

308

309

310

311

312

313

314

315

316

317

318

319

320

321

322

323

324

analyzed the expression of UCPs in the WAT. The data revealed that the mRNA of UCP1 and UCP3 increased markedly, suggesting the induction of WAT browning by RSE (Fig. 5B).

PPARs are the ligand-activated nuclear transcription factors regulating the gene expression of glucose and lipid metabolism. A reporter assay was performed to test whether the RSE alters transactivities of PPAR $\alpha, \beta, \gamma$. The results showed that RSE did not change the transcription activity of PPARs, suggesting that RSE does not activate PPARs directly.

We examined the mRNA expression levels of PPARs and their target genes in the WAT. RSE clearly increased the mRNA expression of PPARs and acetyl coenzyme A carboxylase (ACC), acyl-CoA oxidase (ACO), adipose fatty acid-binding protein (aP2), cluster of differentiation 36 (CD36), peroxisome proliferator-activated receptor coactivator-1 $\alpha$ and $-1 \beta$ (PGC-1 $\alpha$ and PGC$1 \beta$ ), glucose transporter 4 (GLUT 4) as well as UCP1, and UCP3. Among them, PPAR $\beta$, PPAR $\gamma$, $\mathrm{UCP} 1, \mathrm{UCP} 3, \mathrm{ACO}, \mathrm{aP2}$, and CD 36 were significant (Fig. 5B). Taken together, the data suggested that RSE may regulate body weight and blood glucose levels through the enhancement of PPAR signaling.

\section{DISCUSSION}

MS is a complex health problem involving several complications and is prevalent in both developed and developing countries. Host genetic and environmental factors can result in MS (Lim et al., 2016).Wu et al. reported that physical activity can serve as an effective means to prevent metabolic syndrome ( $W u$ et al., 2016). However, physical activity alone does not effectively prevent MS; drugs are also required. Therefore, the development of new drugs to manage MS is necessary.

RS, a common Chinese herbal medicine, has been used in the treatment of deficiency-heat syndrome. Some compounds extracted from RS have different potential pharmacological effects, for instance, saikosaponin C can prevent Alzheimer's disease in various neuronal models and saikosaponin D can inhibit selectin-mediated cell adhesion (Jang et al., 2014). Furthermore, the plant extract of RS is used as a reducing agent to convert gold ions to gold nanoparticles in a biofabrication process. An increasing number applications of RS have been recently discovered, such as prevention of depression and anxiety-like behaviors in rats exposed to repeated restraint stress. However, there was no reported that RS could treat obesity. We should investigated promising new features of Chinese traditional medicine.

Through LC-HRMS, we detected some chemical constituents of RSE that were mainly $\beta$ carboline alkaloids ( $\beta \mathrm{CAs})$ : dichotomine $\mathrm{B}$, dichotomine $\mathrm{H}$, dichotomine $\mathrm{L}$, stellarine $\mathrm{A}-\mathrm{C}$ and glucodichotomine B. $\beta$ CAs are a type of heterocyclic amines; they are considered to be products of cooking meat (Lavita et al., 2015), and widely distributed in the nature and their action is similar to that of indole alkaloids. Recent years, cyclopeptides have received considerable attention from pharmacologists, chemists and biochemists owing to their various bioactivities 
325

326

327

328

329

330

331

332

333

334

335

336

337

338

339

340

341

342

343

344

345

346

347

348

349

350

351

352

353

354

355

356

357

358

359

360

361

362

363

364

365

such as antiviral, antineoplastic, immunomodulate properties. For example, as a main chemical constituent of Psammosilene tunicoides, stallarine A, a new cyclic heptapeptide (Zhao et al., 1995) showed the bacteriostatic activity to a certain extent (Wang et al., 2012). Besides, a study reported that glucodichotomine B and neolignan glycosides isolated from the root of RS showed antiallergic activities (Morikawa et al., 2004). In a word, $\beta$ CAs exhibit anti-tumor, anti-microbial, anti-viral ( $\mathrm{Li}$ et al., 2006), anti-oxidative (Hadjaz et al., 2011) and insecticidal activities. Moreover, according to some previous studies, we found that many alkaloids were identified to have PPARs agonistic activity: picrasidine $\mathrm{C}$ and picrasidine $\mathrm{N}$ (Zhao et al., 2016), isolated from the root of Picrasma quassioides, were identified to have PPAR $\alpha$ and PPAR $\beta$ agonistic activity respectively (Zhao et al., 2016); evodiamine, an indole alkaloid extracted from the Chinese medicine evodia, has been shown to inhibit tumor cell invasion and protect the cardiovascular system through activating PPAR $\gamma$ (Ge et al., 2015). In the present study, $\beta C A s$ constituted a large proportion of the RSE; therefore, we suspect that they played a role in treating obesity through regulating PPARs.

In the present study, RSE treatment significantly reduced body weight and WAT size in the C57BL/6 mice compared with HF group. Weight-reduction therapy involves three major methods: reducing food intake, increasing energy expenditure, and inhibiting lipid absorption. We did not observe significant changes in food consumption and lipid absorption in the intestine. Interestingly, the rectal temperature of RSE-treated mice was markedly higher than that of HFdiet-fed mice. These data suggest that RSE could block body-weight gain by increasing the energy metabolism rather than by reducing calorie intake or inhibition of intestinal lipid absorption. Our findings indicated that RS also could be used to increase the body temperature in obese subjects.

RSE-treated mice exhibited lower fasting blood glucose levels and improved insulin tolerance than the mice in the other groups. Obesity is a crucial risk factor for metabolic disorders, moreover, weight reduction may improve insulin tolerance and diabetes. Therefore, the bodyweight reduction might contributed to the glucose-lowering effects of RSE.

For the mechanism of increasing energy expenditure, we focused on the mRNA expression levels of related genes in BAT and WAT, which play a vital role in energy metabolism. Recently, "beige adipocytes" have been identified (Wu et al., 2012), which share common morphology and function with classical BAT, but they are observed in WAT (Shin et al., 2016). Beige adipocytes can promote browning in WAT and increase the expression levels of UCPs, and efficiently increasing energy expenditure by elevating thermogenesis. Therefore, "beige adipocytes" provide a platform for anti-obesity therapy (Harms \& Seale, 2013; Pfeifer \& Hoffmann, 2015). Our results suggested that RSE might block body weight gain induced by HF-diet in C57BL/6 mice through the elevation of energy metabolism genes expression promoting the white fat beige, evidenced by the high-level expression of UCPs (Nedergaard \& Cannon, 2014). 
According to the experimental results, the expression of PPARs and downstream genes were increased, indicating that RSE may activate PPARs signaling. Uncoupling protein3 (UCP3) is a mitochondrial anion carrier protein, regarded as an obesity candidate gene. It is mainly distributed in the skeletal muscles and BAT, and it is also expressed in WAT. UCP3 could mediate the oxidation process and ADP uncoupling phosphorylation process, thereby preventing energy storage in the form of ATP but releasing it in the form of heat. Evidence supported the role of UCP3 in the lipid metabolic, glucose metabolic(Busiello, Savarese \& Lombardi, 2015), and energy balance of the body, specifically glucose oxidation and insulin sensitivity(Bezaire, Seifert \& Harper, 2007; Busiello, Savarese \& Lombardi, 2015). RSE sharply increased the mRNA levels of PPARs and target gene UCP3, thereby suggesting that RS prevents HF-dietinduced obesity in C57BL/6 mice mainly through the activation of PPARs and UCP3 signaling.

\section{CONCLUSION}

RS may alleviate metabolic disorders, by inhibiting body weight increase, reducing fasting blood glucose levels, and ameliorating insulin tolerance in HF diet-induced obese C57BL/6 mice through the increase of UCP3 and PPARs. Our data suggest that RS may be used to prevent metabolic disorders in addition to its traditional uses. However, the potential effects of RS have yet to be discovered, and the identification of active ingredients and elucidation of mechanisms underlying the alleviation of metabolic disorders call for further inquiry.

\section{ACKNOWLEDGMENTS}

The authors would like to thank F. Li (State Key Laboratory of Natural Medicines, Department of Natural Medicinal Chemistry, China Pharmaceutical University, People's Republic of China) for his useful suggestions and revision.

\section{REFERENCES}

Morikawa T, Sun B, Matsuda H, Wu L, Harima S, Yoshikawa M. 2004. Bioactive constituents from Chinese natural medicines. XIV. New glycosides of beta-carboline-type alkaloid, neolignan, and phenylpropanoid from Stellaria dichotoma L. var. lanceolata and their antiallergic activities. Chem Pharm Bull (Tokyo) 52(10):1194-1199.

Eckel RH, Alberti KG, Grundy SM, Zimmet PZ. 2010. The metabolic syndrome. Lancet 375(9710):181-183.

Ginsberg HN. 2003. Treatment for patients with the metabolic syndrome. Am J Cardiol 91(7A):29E-39E.

Apovian CM, Aronne L, Bessesen DH, McDonnell ME, Murad MH, Pagotto U, Ryan DH, Still CD. 2015. Pharmacological management of obesity: an endocrine Society clinical practice guideline. J Clin Endocrinol Metab 100(2):342-362.

Harms M, Seale P. 2013. Brown and beige fat: development, function and therapeutic potential. Nat Med 19(10):1252-1263.

Izzi-Engbeaya C, Salem V, Atkar RS, Dhillo WS. 2015. Insights into Brown Adipose Tissue Physiology as Revealed by 
Imaging Studies. Adipocyte 4(1):1-12.

Kim SH, Plutzky J. 2016. Brown Fat and Browning for the Treatment of Obesity and Related Metabolic Disorders. Diabetes Metab J 40(1):12-21.

Francis GA, Fayard E, Picard F, Auwerx J. 2003. Nuclear receptors and the control of metabolism. Annu Rev Physiol 65(261-311.

Desvergne B, Wahli W. 1999. Peroxisome proliferator-activated receptors: nuclear control of metabolism. Endocr Rev 20(5):649-688.

Wahli W, Braissant O, Desvergne B. 1995. Peroxisome proliferator activated receptors: transcriptional regulators of adipogenesis, lipid metabolism and more. Chem Biol 2(5):261-266.

Jia Y, Qi C, Zhang Z, Hashimoto T, Rao MS, Huyghe S, Suzuki Y, Van Veldhoven PP, Baes M, Reddy JK. 2003. Overexpression of peroxisome proliferator-activated receptor-alpha (PPARalpha)-regulated genes in liver in the absence of peroxisome proliferation in mice deficient in both L- and D-forms of enoyl-CoA hydratase/dehydrogenase enzymes of peroxisomal beta-oxidation system. J Biol Chem 278(47):4723247239.

Barger PM, Kelly DP. 2000. PPAR signaling in the control of cardiac energy metabolism. Trends Cardiovasc Med 10(6):238-245.

Gilde AJ, Van Bilsen M. 2003. Peroxisome proliferator-activated receptors (PPARS): regulators of gene expression in heart and skeletal muscle. Acta Physiol Scand 178(4):425-434.

Bishop-Bailey D. 2000. Peroxisome proliferator-activated receptors in the cardiovascular system. Br J Pharmacol 129(5):823-834.

Puddu P, Puddu GM, Muscari A. 2003. Peroxisome proliferator-activated receptors: are they involved in atherosclerosis progression? Int J Cardiol 90(2-3):133-140.

Thompson EA. 2007. PPARgamma physiology and pathology in gastrointestinal epithelial cells. Mol Cells 24(2):167176.

Spiegelman BM. 1998. PPAR-gamma: adipogenic regulator and thiazolidinedione receptor. Diabetes 47(4):507-514. Barroso I, Gurnell M, Crowley VE, Agostini M, Schwabe JW, Soos MA, Maslen GL, Williams TD, Lewis H, Schafer AJ, Chatterjee VK, O'Rahilly S. 1999. Dominant negative mutations in human PPARgamma associated with severe insulin resistance, diabetes mellitus and hypertension. Nature 402(6764):880-883.

Chen YF, Kuo PC, Chan HH, Kuo IJ, Lin FW, Su CR, Yang ML, Li DT, Wu TS. 2010. beta-carboline alkaloids from Stellaria dichotoma var. lanceolata and their anti-inflammatory activity. J Nat Prod 73(12):1993-1998.

Cao LH, Zhang W, Luo JG, Kong LY. 2012. Five New $\beta$-Carboline-Type Alkaloids from Stellaria dichotoma var. lanceolata. ChemInform 43(40):

Teng J. 1985. [Research on the originol plant Stellaria dichotoma var. Lanceolata in the ben-cao pharmaopeia]. Zhong Yao Tong Bao 10(4):15-16.

Sun B, Morikawa T, Matsuda H, Tewtrakul S, Wu L, Harima S, Yoshikawa M. 2004. Structures of new betacarboline-type alkaloids with antiallergic effects from Stellaria dichotoma(1,2). J Nat Prod 67(9):14641469.

Morita H, lizuka T, Choo CY, Chan KL, Itokawa H, Takeya K. 2005. Dichotomins J and K, vasodilator cyclic peptides from Stellaria dichotoma. J Nat Prod 68(11):1686-1688.

Sun H, Wang X, Chen J, Song K, Gusdon AM, Li L, Bu L, Qu S. 2016. Melatonin improves non-alcoholic fatty liver disease via MAPK-JNK/P38 signaling in high-fat-diet-induced obese mice. Lipids Health Dis 15(1):202. 
Xia SF, Le GW, Wang P, Qiu YY, Jiang YY, Tang X. 2016. Regressive Effect of Myricetin on Hepatic Steatosis in Mice Fed a High-Fat Diet. Nutrients 8(12):

Zhang, DF. 2002. Experiments of pharmacology and traditional Chinese medicine pharmacology . Shanghai: Shanghai Scientific and Technical Publishers, 8.

Nedergaard J, Cannon B. 2014. The browning of white adipose tissue: some burning issues. Cell Metab 20(3):396407.

Lim MY, You HJ, Yoon HS, Kwon B, Lee JY, Lee S, Song YM, Lee K, Sung J, Ko G. 2016. The effect of heritability and host genetics on the gut microbiota and metabolic syndrome. Gut

Wu S, Fisher-Hoch SP, Reininger B, McCormick JB. 2016. Recommended Levels of Physical Activity Are Associated with Reduced Risk of the Metabolic Syndrome in Mexican-Americans. PLoS One 11(4):e0152896.

Jang MJ, Kim YS, Bae EY, Oh TS, Choi HJ, Lee JH, Oh HM, Lee SW. 2014. Saikosaponin D isolated from Bupleurum falcatum inhibits selectin-mediated cell adhesion. Molecules 19(12):20340-20349.

Lavita SI, Aro R, Kiss B, Manto M, Duez P. 2015. The Role of beta-Carboline Alkaloids in the Pathogenesis of Essential Tremor. Cerebellum

Zhao YR, Zhou J, Wang XK, Huang XL, Wu HM, Zou C. 1995. Cyclopeptides from Stellaria yunnanensis. Phytochemistry 40(5):1453-1456.

Wang L, Gong XJ, Zhou X, Xian C, Yang SL, Yang ZN. 2012. [Chemical constituents of Psammosilene tunicoides and bacteriostatic activity]. Zhongguo Zhong Yao Za Zhi 37(23):3577-3580.

Li XL, Cheng X, Yang LM, Wang RR, Zheng YT, Xiao WL, Zhao Y, Xu G, Lu Y, Chang Y, Zheng QT, Zhao QS, Sun HD. 2006. Dichotomains $A$ and $B$ : two new highly oxygenated phenolic derivatives from Dicranopteris dichotoma. Org Lett 8(9):1937-1940.

Hadjaz F, Besret S, Martin-Nizard F, Yous S, Dilly S, Lebegue N, Chavatte P, Duriez P, Berthelot P, Carato P. 2011. Antioxydant activity of beta-carboline derivatives in the LDL oxidation model. Eur J Med Chem 46(6):25752585.

Zhao S, Kanno Y, Li W, Wakatabi H, Sasaki T, Koike K, Nemoto K, Li H. 2016. Picrasidine N Is a Subtype-Selective PPARbeta/delta Agonist. J Nat Prod 79(4):879-885.

Zhao S, Kanno Y, Li W, Sasaki T, Zhang X, Wang J, Cheng M, Koike K, Nemoto K, Li H. 2016. Identification of Picrasidine C as a Subtype-Selective PPARalpha Agonist. J Nat Prod 79(12):3127-3133.

Ge X, Chen S, Liu M, Liang T, Liu C. 2015. Evodiamine Attenuates PDGF-BB-Induced Migration of Rat Vascular Smooth Muscle Cells through Activating PPARgamma. Int J Mol Sci 16(12):28180-28193.

Wu J, Bostrom P, Sparks LM, Ye L, Choi JH, Giang AH, Khandekar M, Virtanen KA, Nuutila P, Schaart G, Huang K, Tu H, van Marken Lichtenbelt WD, Hoeks J, Enerback S, Schrauwen P, Spiegelman BM. 2012. Beige adipocytes are a distinct type of thermogenic fat cell in mouse and human. Cell 150(2):366-376.

Shin JH, Lee SH, Kim YN, Kim IY, Kim YJ, Kyeong DS, Lim HJ, Cho SY, Choi J, Wi YJ, Choi JH, Yoon YS, Bae YS, Seong JK. 2016. AHNAK deficiency promotes browning and lipolysis in mice via increased responsiveness to betaadrenergic signalling. Sci Rep 6(23426.

Pfeifer A, Hoffmann LS. 2015. Brown, beige, and white: the new color code of fat and its pharmacological implications. Annu Rev Pharmacol Toxicol 55(207-227.

Busiello RA, Savarese S, Lombardi A. 2015. Mitochondrial uncoupling proteins and energy metabolism. Front Physiol 6(36.

Bezaire V, Seifert EL, Harper ME. 2007. Uncoupling protein-3: clues in an ongoing mitochondrial mystery. FASEB J 


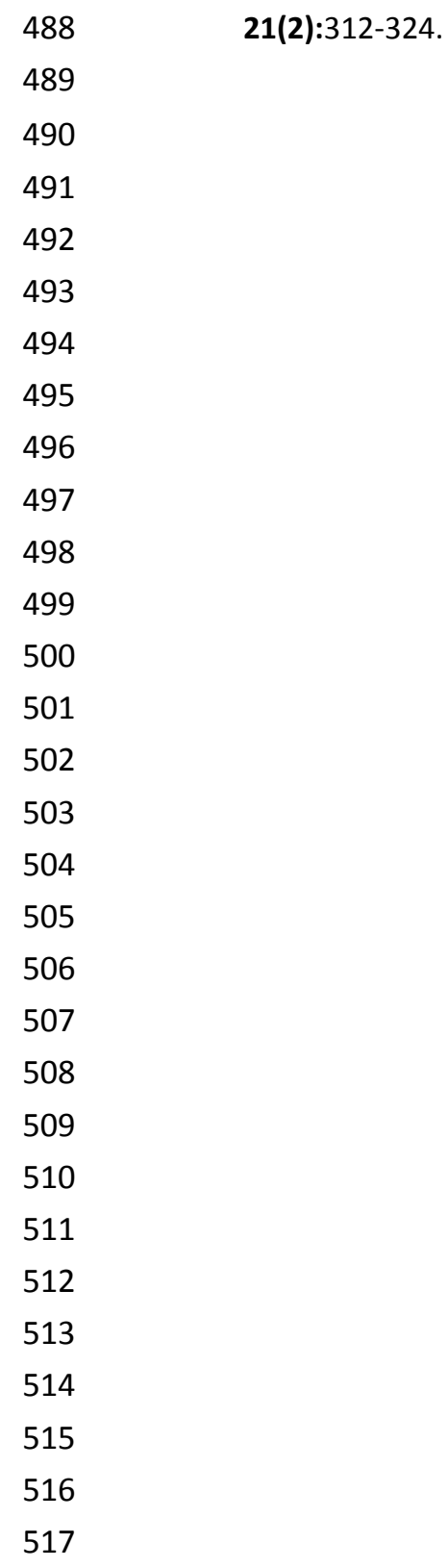

513

514

515

516

517

518

$519 \quad$ Fig 1 

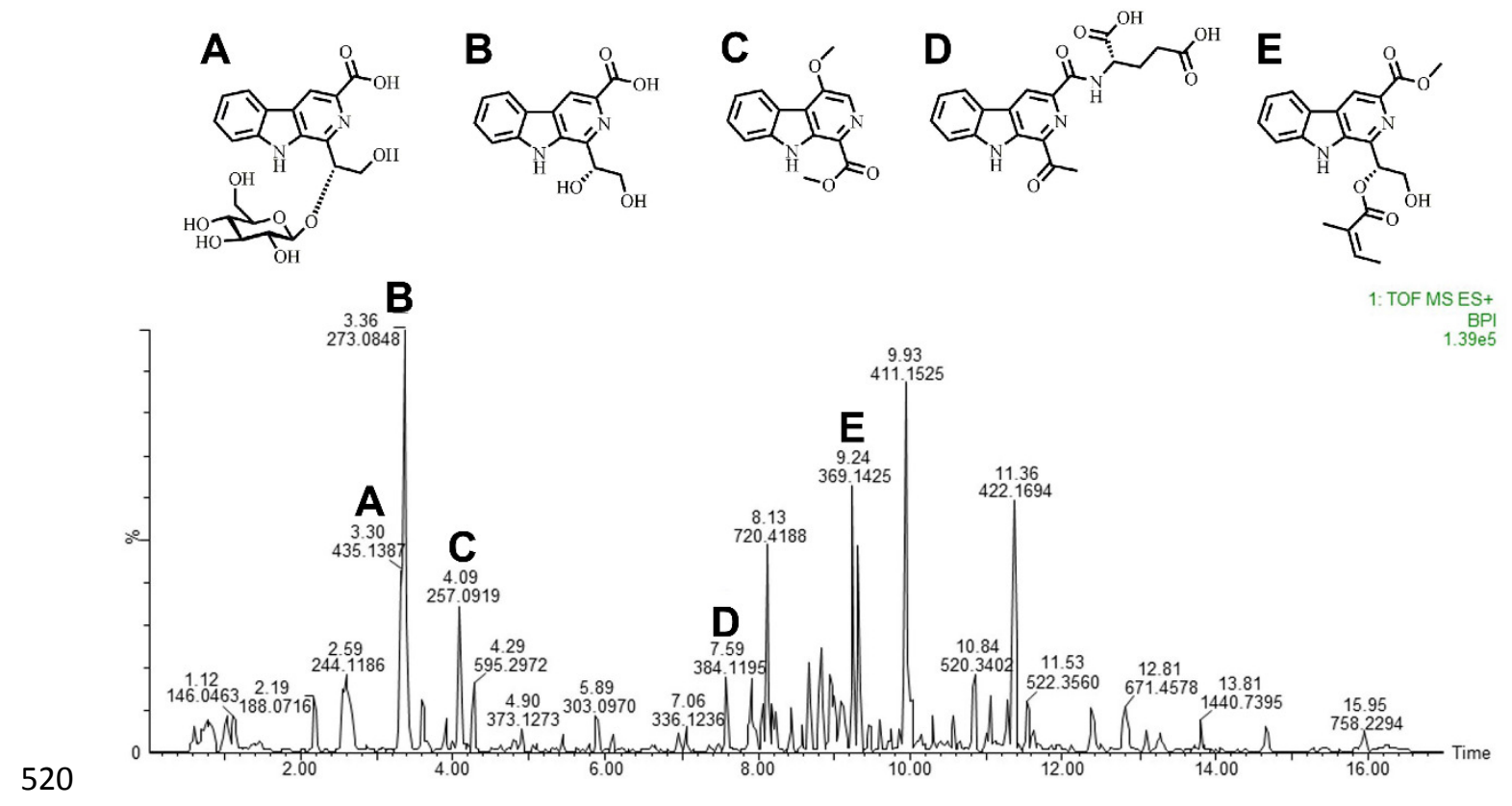

521

Carbolines of RSE identified through liquid chromatography-high resolution mass spectrometry. Total ion chromatogram of the chemical composition in RSE identified through (C) $\beta$-carboline alkaloid (D)Dichotomine H (E) Dichotomine L

526

527

528

529

530

531

532

533

534

535

536

537

538

539

540 
547 Fig 2

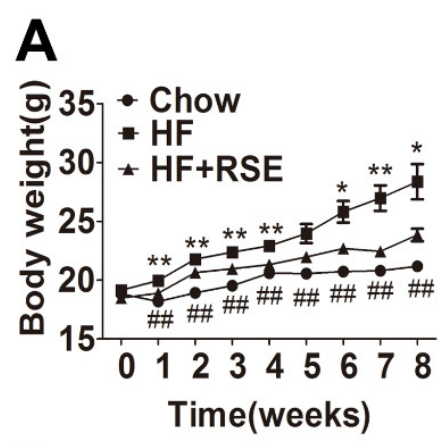

D Chow

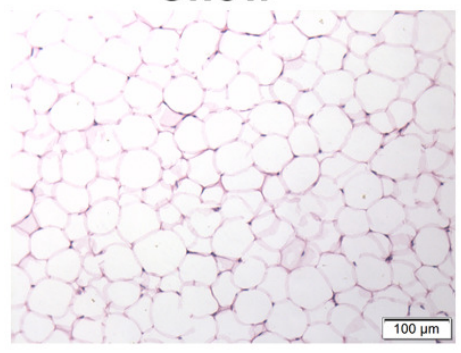

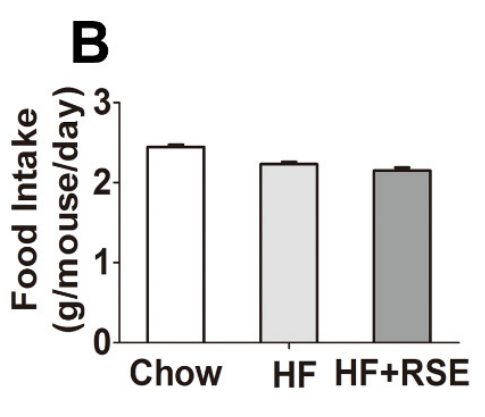

$\mathrm{HF}$

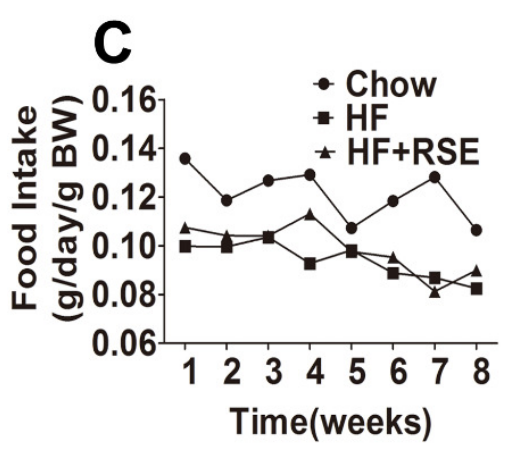

HF+RSE
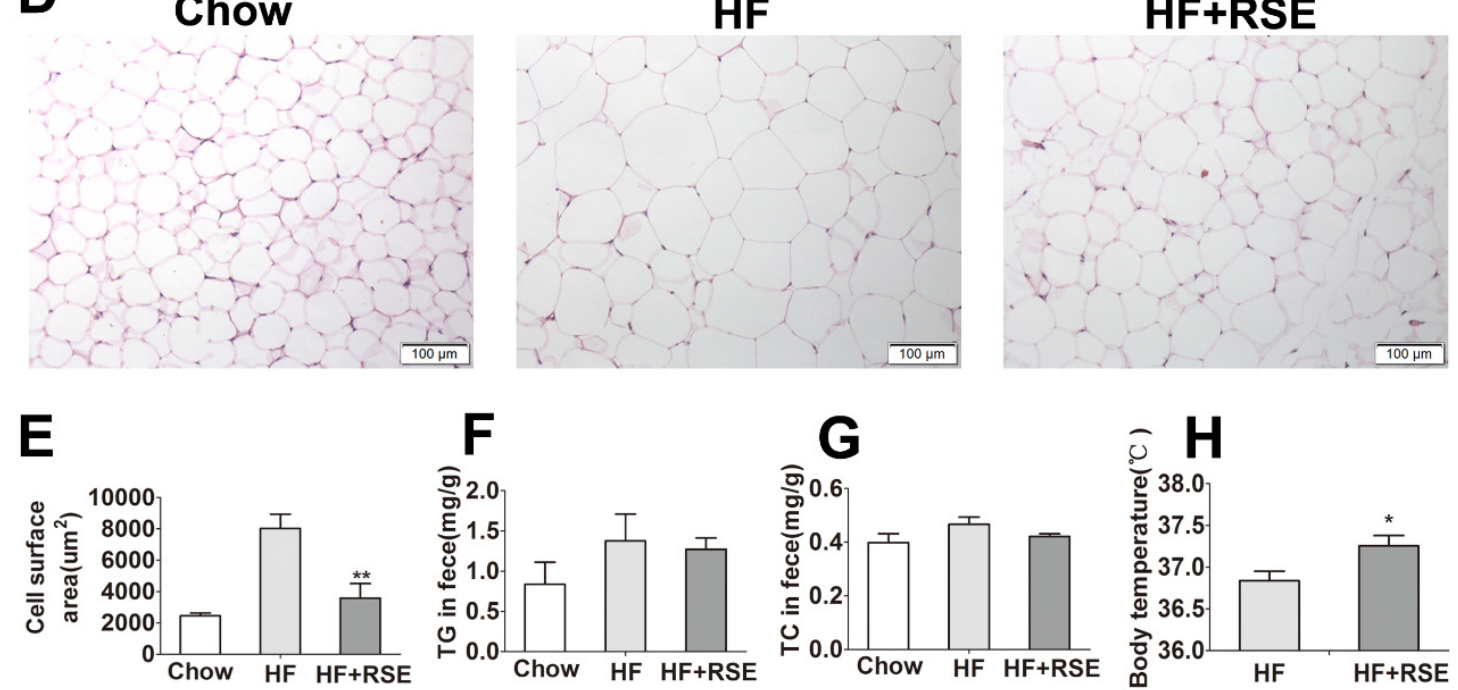

548

RSE prevents metabolic disorders in high-fat-diet -induced obese C57BL/6 mice. The mice were fed with Chow, HF-diet, and HF-diet mixed with $1 \%(\mathrm{w} / \mathrm{w})$ RSE for 8 weeks. (A) Body weight (B) Food intake amount (C) Food intake dynamic figure (D) H\&E staining of WAT sections $(200 \times)($ E) Cell surface area of WAT $(F)$ Feces TG levels $(G)$ Feces TC levels $(H)$ Body temperature. Data are presented as mean \pm SEM (Chow: $\mathrm{n}=10$; others: $\mathrm{n}=8$ ). $* P<0.05$, ${ }^{* *} P<0.01$ vs the HF group. ${ }^{\#} P<0.05$, ${ }^{\#} P<0.01$ were the Chow vs the HF group. 
565

566

567

568

569

570

571

572

573

574

575

576

577

578

579

580

581

582

583

584

585

586

587

588

589

590

591

592

593

594

595

596

84

86

87

90

92

3

Fig 3
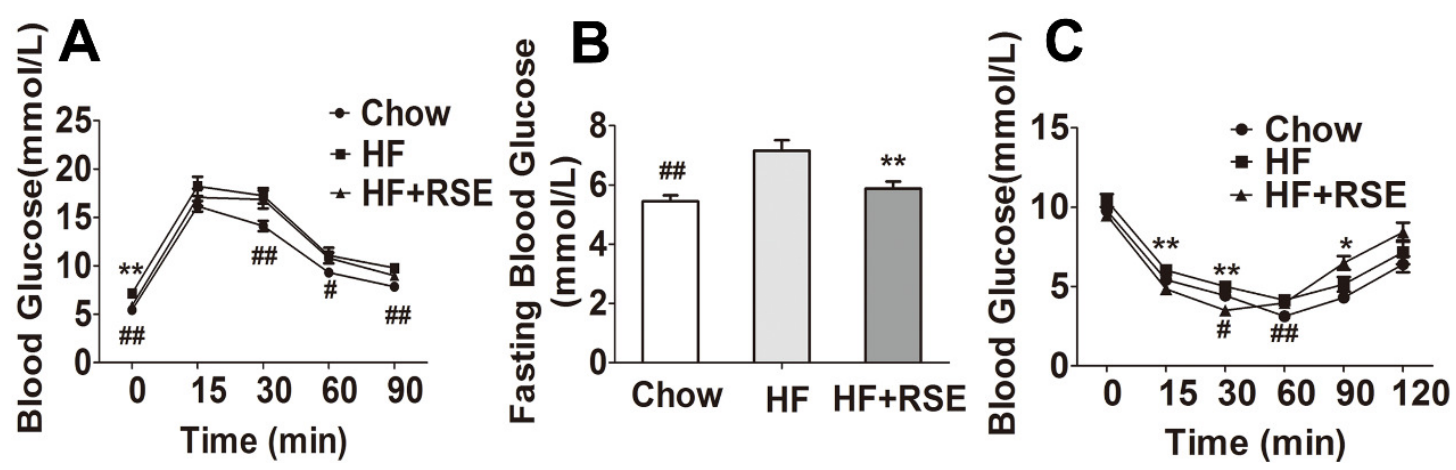

RSE improves glucose metabolism and insulin tolerance in high-fat-diet-induced C57BL/6 mice. (A) Intraperitoneal glucose tolerance test at 0, 15, 30, 60, and $90 \mathrm{~min}$. The mice were fasted for $12 \mathrm{~h}$ before measuring blood glucose levels at 0 min (B) Fasting glucose levels (C) Intraperitoneal insulin tolerance test at $0,15,30,60,90$, and $120 \mathrm{~min}$. The mice were not fasted. Data are presented as mean \pm SEM (Chow: $\mathrm{n}=10$; others: $\mathrm{n}=8$ ). ${ }^{*} P<0.05$, $* * P<0.01$ vs the

HF group. ${ }^{\#} P<0.05,{ }^{\# \#} P<0.01$ were the Chow vs the HF group.

列

8

9

1

3

88

1


Fig 4
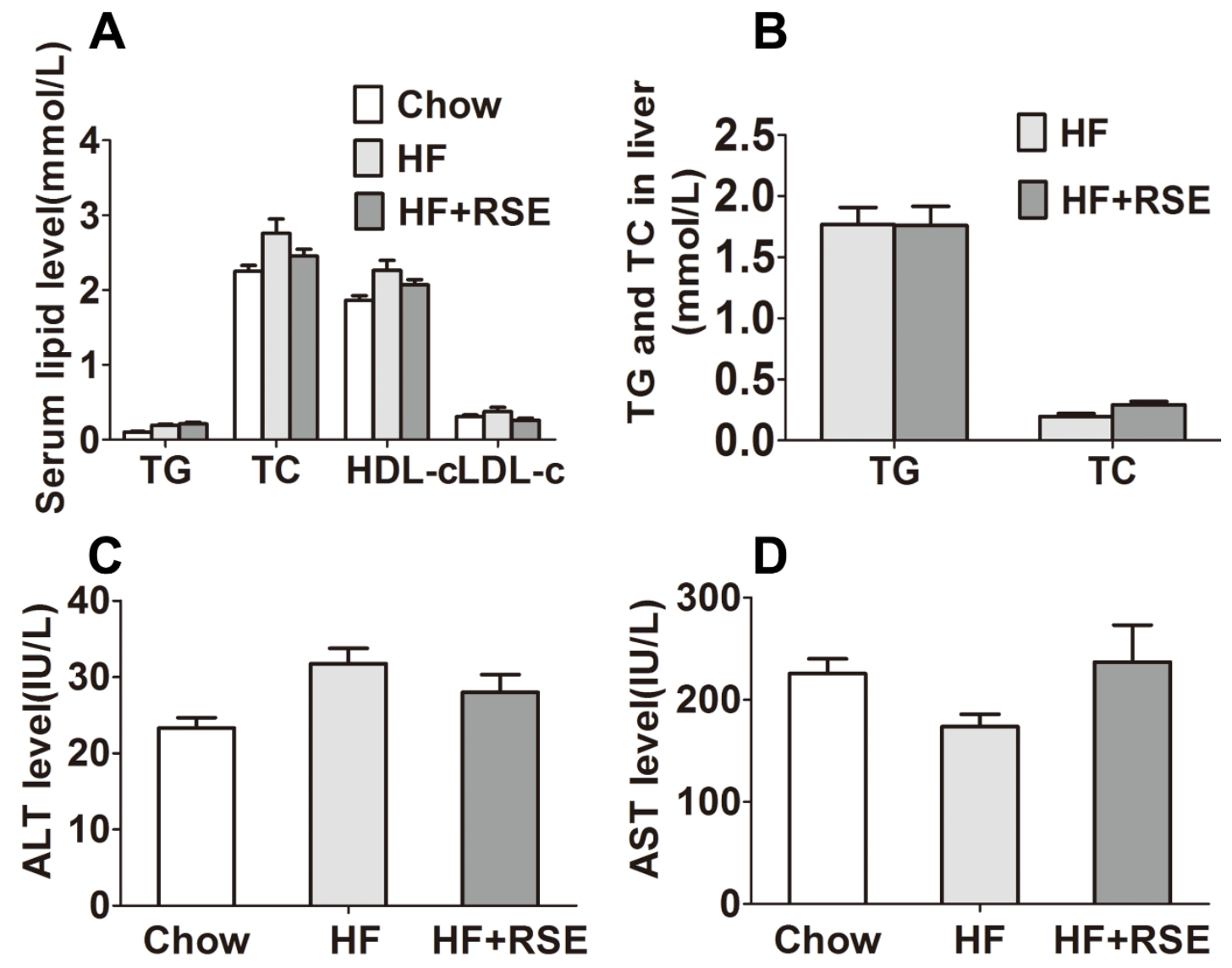

603

604

605

606

607

608

609

610

611

612

613

614

615

616

Effects of RSE on serum and liver lipid levels in high-fat-diet-induced C57BL/6 mice. (A) Serum TC, TG, HDL-c, LDL-c levels (B) Liver TG and TC levels (C) ALT levels in serum (D) AST levels in serum. Data are presented as mean \pm SEM (Chow: $n=10$; others: $n=8$ ), Figure B comparison between group pairs were K-W H test because of the data not following normal distribution. $* P<0.05, * * P<0.01$ vs the HF group. 
Fig 5

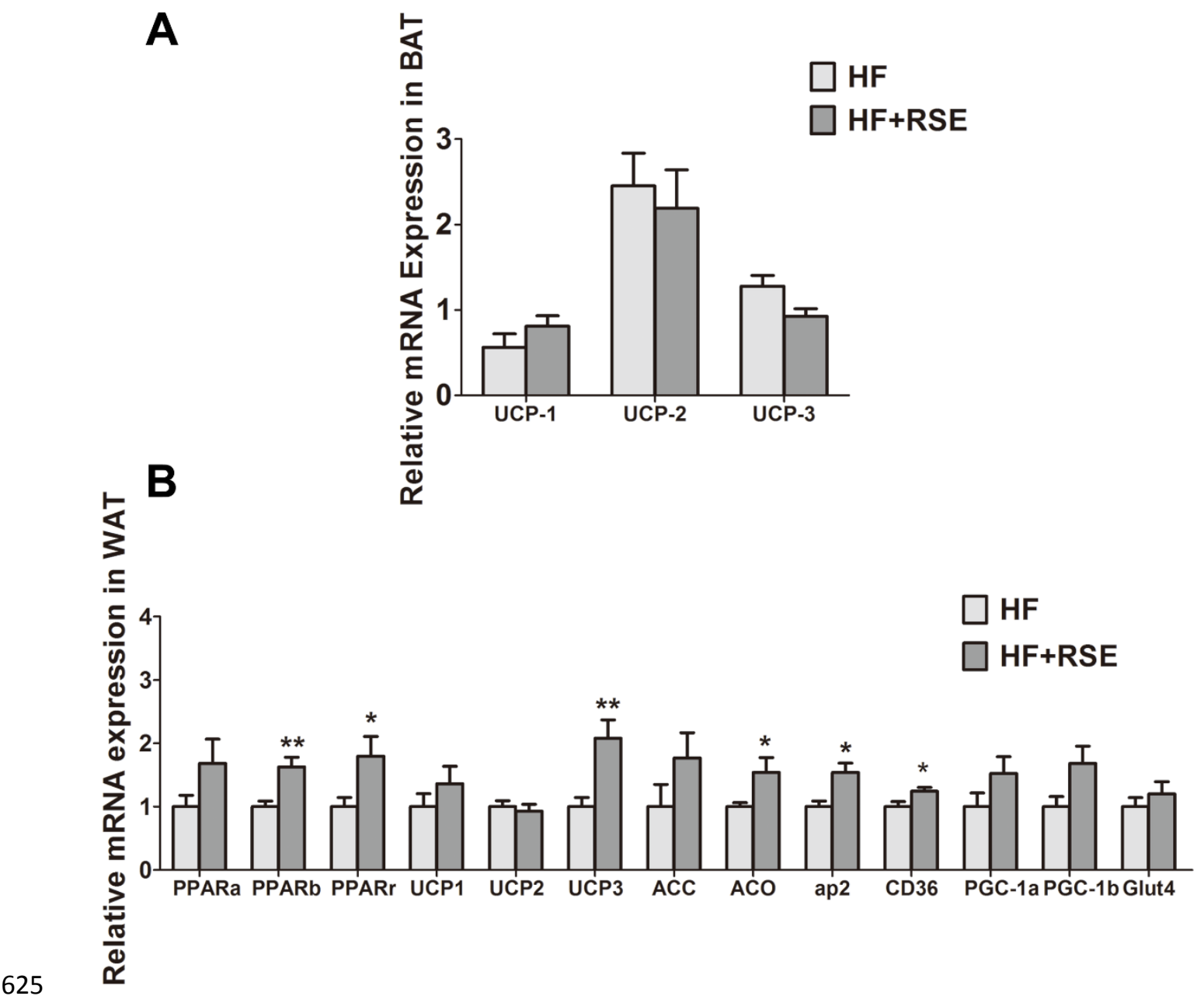

RSE induced the expression of UCPs, PPARs and their target genes. (A) Relative expression $<0.01$ vs the HF group. 
635
636
637
638
639
640
641
642
643
644
$645 \quad$ Table 1: Sequences of the primers used in real-time PCR of mouse tissue.

\begin{tabular}{|c|c|c|}
\hline Gene & Forward primer & Reverse primer \\
\hline$\beta$-Actin & TGTCCACCTTCCAGCAGATGT & AGCTCAGTAACAGTCCGCCTAGA \\
\hline \multirow[t]{2}{*}{$\operatorname{PPAR} \alpha$} & AGGCTGTAAGGGCTTCTTTC & GGCATTTGTTCCGGTTCTTC \\
\hline & G & \\
\hline PPAR $\beta$ & AGTGACCTGGCGCTCTTCAT & CGCAGAATGGTGTCCTGGAT \\
\hline $\operatorname{PPAR} \gamma$ & CGCTGATGCACTGCCTATGA & AGAGGTCCACAGAGCTGATTCC \\
\hline PGC-1 $\alpha$ & TGTTCCCGATCACCATATTCC & GGTGTCTGTAGTGGCTTGATTC \\
\hline \multirow[t]{2}{*}{ PGC-1 $\beta$} & GGGTGCGCCTCCAAGTG & TCTACAGACAGAAGATGTTATGT \\
\hline & & GAACAC \\
\hline $\mathrm{aP} 2$ & CATGGCCAAGCCCAACAT & CGCCCAGTTTGAAGGAAATC \\
\hline \multirow[t]{2}{*}{$\mathrm{ACC}$} & GAATCTCCTGGTGACAATGC & GGTCTTGCTGAGTTGGGTTAGCT \\
\hline & TTATT & \\
\hline $\mathrm{ACO}$ & CAGCACTGGTCTCCGTCATG & CTCCGGACTACCATCCAAGATG \\
\hline UCP1 & CATCACCACCCTGGCAAAA & AGCTGATTTGCCTCTGAATGC \\
\hline $\mathrm{UCP} 2$ & GGGCACTGCAAGCATGTGTA & TCAGATTCCTGGGCAAGTCACT \\
\hline
\end{tabular}




\begin{tabular}{|c|c|c|c|c|c|c|c|}
\hline \multicolumn{2}{|c|}{ UCP3 } & \multicolumn{3}{|c|}{ TGGCCCAACATCACAAGAAA } & \multicolumn{3}{|c|}{ TCCAGCAACTTCTCCTTGATGA } \\
\hline \multicolumn{2}{|c|}{ CD36 } & \multicolumn{3}{|c|}{ GCTTGCAACTGTCAGCACAT } & \multicolumn{3}{|c|}{ GCCTTGCTGTAGCCAAGAAC } \\
\hline \multicolumn{2}{|c|}{ Glut4 } & \multicolumn{3}{|c|}{ GTAACTTCATTGTCGGCATG } & \multicolumn{3}{|c|}{ AGCTGAGATCTGGTCAAACG } \\
\hline & & \multicolumn{6}{|l|}{$\mathrm{G}$} \\
\hline \multicolumn{8}{|l|}{646} \\
\hline \multicolumn{8}{|l|}{647} \\
\hline \multicolumn{8}{|l|}{648} \\
\hline \multicolumn{8}{|l|}{649} \\
\hline \multicolumn{8}{|l|}{650} \\
\hline \multicolumn{8}{|l|}{651} \\
\hline \multicolumn{8}{|l|}{652} \\
\hline \multicolumn{8}{|l|}{653} \\
\hline \multicolumn{8}{|l|}{654} \\
\hline \multirow{3}{*}{656} & Table & 2: Chem & cal constituents & f RSE iden & ified thr & ugh liquid ch & omatography-high resolution mass \\
\hline & & rometry. & & & & & \\
\hline & Peak & $\operatorname{Rt}(\min )$ & MS-Mol. wt. $+\mathrm{H}$ & Actual Mo & . wt. $+\mathrm{H}$ & Formula & Constituents \\
\hline & 1 & 3.30 & 435.1387 & 435.1359 & & $\mathrm{C}_{20} \mathrm{H}_{22} \mathrm{~N}_{2} \mathrm{O}_{9}$ & Glucodichotomine B \\
\hline & 2 & 3.362 & 273.0850 & 273.0875 & & $\mathrm{C}_{14} \mathrm{H}_{12} \mathrm{~N}_{2} \mathrm{O}_{4}$ & Dichotomine B \\
\hline & 3 & 4.070 & 257.0919 & 257.0926 & & $\mathrm{C}_{14} \mathrm{H}_{12} \mathrm{~N}_{2} \mathrm{O}_{3}$ & $\beta$-carboline alkaloid \\
\hline & 4 & 7.59 & 384.1195 & 384.1151 & & $\mathrm{C}_{19} \mathrm{H}_{17} \mathrm{~N}_{3} \mathrm{O}_{6}$ & Dichotomine $\mathrm{H}$ \\
\hline & 5 & 9.238 & 369.1425 & 369.1450 & & $\mathrm{C}_{20} \mathrm{H}_{20} \mathrm{~N}_{2} \mathrm{O}_{5}$ & Dichotomine L \\
\hline & 6 & 7.614 & 254.0919 & 254.0930 & & $\mathrm{C}_{14} \mathrm{H}_{11} \mathrm{~N}_{3} \mathrm{O}_{2}$ & Stellarine A \\
\hline & 7 & 8.190 & 338.1138 & 338.1141 & & $\mathrm{C}_{18} \mathrm{H}_{15} \mathrm{~N}_{3} \mathrm{O}_{4}$ & Stellarine B \\
\hline & 8 & 8.940 & 269.0920 & 269.0926 & & $\mathrm{C}_{15} \mathrm{H}_{12} \mathrm{~N}_{2} \mathrm{O}_{3}$ & Stellarine C \\
\hline & 9 & 3.793 & 153.0562 & 153.0552 & & $\mathrm{C}_{8} \mathrm{H}_{8} \mathrm{O}_{3}$ & Vanillin \\
\hline & 10 & 2.058 & 127.0392 & 127.0395 & & $\mathrm{C}_{6} \mathrm{H}_{6} \mathrm{O}_{3}$ & 5-Hydroxymethylfurfural \\
\hline
\end{tabular}


657 Rt: retention time (min), MS-Mol. wt.+H: primary mass spectrometry, Actual Mol. wt.+H: actual molecular 658 weight.

659

660 